\title{
MODULES WITH ABELIAN ENDOMORPHISM RINGS
}

\author{
GRIGORE CĂLUGĂREANU and PHILL SCHULTZ ${ }^{凶}$
}

(Received 15 October 2009)

\begin{abstract}
The results of Szele and Szendrei ['On Abelian groups with commutative endomorphism rings', Acta Math. Acad. Sci. Hungar. 2 (1951), 309-324] characterizing abelian groups with commutative endomorphism rings are generalized to modules whose endomorphism rings have various restrictions on their idempotents. Such properties include central or commuting idempotents, and one-sided ideals being two-sided. Related properties include direct summands having unique complements, or being fully invariant.
\end{abstract}

2000 Mathematics subject classification: primary 16D70; secondary 16D99.

Keywords and phrases: commutative endomorphism rings, central or commuting idempotents, duo rings, unique complements, fully invariant submodules.

\section{Introduction}

Throughout this paper, unless specifically noted, the word ring denotes a unital associative ring, group denotes an abelian group, and module denotes a unital right $R$-module. It is a long-standing problem in abelian group theory to describe those groups $G$ whose endomorphism $\operatorname{ring} \operatorname{End}(G)$ is commutative and those commutative rings which are isomorphic to the endomorphism ring of an abelian group. Krylov et al. [KMT03] present an authoritative survey of the history of the problems. The known results are of two sorts: if $G$ is characterized by torsion, then a complete classification is known for both parts of the problem [KMT03, Corollary 19.3]; if $G$ is torsion-free, then no classification is feasible; instead, there are realization theorems stating that large classes of commutative rings can be realized as $\operatorname{End}(G)$ for some group $G$ [KMT03, Section 29]. Naturally, mixed groups lie between these two extremes.

The purpose of this paper is to generalize the problem by considering modules whose endomorphism ring is 'almost commutative'. For example, we describe classes of modules whose endomorphism rings have commuting idempotents, or in which one-sided ideals are two-sided. We also consider modules for which direct summands have unique complements or are fully invariant.

(C) 2010 Australian Mathematical Publishing Association Inc. 0004-9727/2010 \$16.00 
Let us first establish some notation. Elements of a ring or module are denoted by lower-case Latin letters, and homomorphisms by lower-case Greek letters. Functions are written on the left of their arguments. We denote a submodule $N$ of $M$ by $N \leq M$ and a direct summand $N$ of $M$ by $N \leq_{\oplus} M$. In particular, if $R$ is a ring, then $I \leq R$ means that $I$ is a right ideal of $R$. We denote the (not necessarily abelian) group of units of $R$ by $U=U(R)$ and the centre of a ring $R$ by $Z=Z(R)$.

Let $R$ be a ring and let $E=E(R)$ denote the set of idempotents in $R$. If $e \in E$, then $e$ is called proper if $e \neq 0$ or 1 . There is a natural partial order, denoted $\leq$, on $E$ defined by $e \leq f$ if $e f=f e=e$, and nonzero minimal elements of $E$ with respect to this order are called primitive idempotents. A ring $R$ is called abelian if $E(R) \subseteq Z(R)$, that is, if all idempotents are central.

If $e \in E$, then $\bar{e}=1-e \in E$ is called the complementary idempotent and $e \bar{e}=$ $\bar{e} e=0$. In general, $e$ and $f \in E$ are called orthogonal if $e f=f e=0$. If $e \in E$ is proper, then $R=e R \oplus \bar{e} R$ is a proper direct decomposition of $R$ considered as right $R$-module. In particular, $e$ is primitive if and only if $e R$ is a minimal right ideal summand. Similar remarks hold of course for left modules $R e$ and $R \bar{e}$. If $e \in E$ is central, then so is $\bar{e}$ and the decomposition is into a direct sum of ideals.

For right $R$-modules $M$ and $N, \operatorname{Hom}(M, N)=\operatorname{Hom}_{R}(M, N)$ denotes the additive group of $R$-homomorphisms of $M$ into $N$ and $\operatorname{End}(M)=\operatorname{End}_{R}(M)$ the ring of $R$-endomorphisms of $M$. The following facts concerning idempotents $\varepsilon \in \operatorname{End}(M)$ are well known [F73, Section 106]. If $\varepsilon$ is a proper idempotent then $\varepsilon$ determines a nontrivial direct decomposition $M=\operatorname{im}(\varepsilon) \oplus \operatorname{ker}(\varepsilon)$. Conversely, if $M=N \oplus K$ is a decomposition of $M$ into nonzero summands, then there is a unique idempotent $\varepsilon \in \operatorname{End}(M)$ such that $\left.\varepsilon\right|_{N}$ is the identity map and $\left.\varepsilon\right|_{K}$ is the zero map. We say that $N \oplus K$ is the decomposition corresponding to $\varepsilon$ and that $\varepsilon$ is the idempotent corresponding to the decomposition $M=N \oplus K$.

The paper is organized as follows. In Section 2, we describe the rings in which direct summand right ideals are uniquely complemented and relate this property to subcommutativity and certain one-sided ideals being two-sided. In Section 3 we show that these properties are equivalent to $R$ being abelian.

In the rest of the paper, the results of Sections 2 and 3 are applied to endomorphism rings of modules. For example, in Section 4 we show how unique complementation of summands of $\operatorname{End}(M)$ implies unique complementation of summands of $M$. We also find a module theoretic generalization of the main result of Szele and Szendrei [SzSz51] on abelian groups with commutative endomorphism rings. Section 5 is devoted to characterizations of the fully invariant submodules and direct summands of a module.

\section{Uniquely complemented summands of rings}

Suppose that $R=I \oplus J$ is a nontrivial decomposition of the ring $R$ into right ideals. Then the idempotent endomorphisms considered above can be realized by multiplication by elements of $E$, the set of idempotents of $R$. In this section, 
we examine the connection between the centrality of $e \in E$ and uniqueness of complements of the direct summand $e R$. We begin with some known facts and some elementary results.

PROPOSITION 2.1 (Folklore). Let $R_{R}=I \oplus J$ be a right ideal direct decomposition of $R$. There exists $e \in E$ such that $I=e R$ and $J=\bar{e} R$.

Much is known about idempotents $e$ and $f$ for which $e R=f R$. For example, here is a selection from Lam [Lam95, Section 21].

PROPOSITION 2.2. Let $e$ and $f \in E$. The following are equivalent:

(1) $e R=f R$;

(2) $e f=f$ and $f e=e$;

(3) there exists $r \in R$ such that $f=e+e r \bar{e}$;

(4) there exists $u \in U$ such that $f=e u$;

(5) $R \bar{e}=R \bar{f}$.

The direct complements of a right ideal direct summand may be characterized as follows.

Lemma 2.3. Let $R=e R \oplus g R$. Then $g R=(\bar{e}-$ er $\bar{e}) R$ for a suitable $r \in R$. Conversely, for every $r \in R,(\bar{e}-$ er $\bar{e}) R$ is a direct complement for $e R$.

PROOF. According to Proposition 2.1, there is an idempotent $f$ such that $e R=f R$ and $g R=\bar{f} R$. By Proposition 2.2, $f=e+e r \bar{e}$ and $g=\bar{f}+\bar{f} s f$ for suitable $r, s \in R$. Thus $g=\bar{f}(1+\bar{f} s f)$ and $u=1+\bar{f} s f$ is invertible in $R$ with inverse $1-\bar{f} s f$. Hence $g=(\bar{e}-e r \bar{e}) u$ so that $g R=(\bar{e}-e r \bar{e}) R$.

As for the converse, again by Proposition 2.1, eR $\oplus(\bar{e}-e r \bar{e}) R=R$ if there is an idempotent $f \in R$ such that $e R=f R$ and $(\bar{e}-e r \bar{e}) R=\bar{f} R$. Clearly $f=e+e r \bar{e}$ is suitable for this.

Since $e R$ always has the direct complement $\bar{e} R$, we say that $e R$ has a unique complement if $R=e R \oplus g R$ implies that $\bar{e} R=g R$.

PROPOSITION 2.4. $e R$ has a unique complement if and only if $e R_{\bar{e}}=0$.

PROOF. Since by Lemma 2.3 all the direct complements of $e R$ are of the form $(\bar{e}-e r \bar{e}) R, e R \bar{e}=0$ implies that $e R$ has a unique complement.

Conversely, if for all $r \in R,(\bar{e}-e r \bar{e}) R=\bar{e} R$, it follows from Proposition 2.2(2) that $(\bar{e}-e r \bar{e}) \bar{e}=\bar{e}$ and so $e r \bar{e}=0$ for all $r \in R$.

Similarly, $e R$ is the unique direct complement of $\bar{e} R$ if and only if $\bar{e} R e=0$.

We now consider the question of when not just the complement of $e R$ but even its idempotent generator $\bar{e}$ is unique.

Proposition 2.5. Let $R=e R \oplus f R$ with $e, f \in E$. Then $f=\bar{e}$ if and only if $e f=f e$. 
PROOF. If $f=\bar{e}$ then $e f=e(1-e)=(1-e) e=f e$.

Conversely, since $e f=f e, \bar{e} f=f \bar{e}$ so that $e f R$ and $\bar{e} f R$ are both contained in $f R$. Decompose $f R$ as $f R=e f R \oplus \bar{e} f R$. Since $e f R \subseteq e R \cap f R=0$, ef $=0$ so that $f R=\bar{e} f R \subseteq \bar{e} R$. Similarly, $\bar{e} R \subseteq f R$ so that $\bar{e} R=f R$.

It follows that $\bar{e}=f r$ for some $r \in R$ and $f=\bar{e} s$ for some $s \in R$. Hence $f \bar{e}=$ $f^{2} r=f r=\bar{e}$ and similarly $\bar{e} f=f$. By commutativity, $\bar{e}=f$.

A similar property concerns commuting idempotent generated right ideals of $R$.

Proposition 2.6. Let $e, f \in E$ satisfy $R=e R \oplus f R$. If $\bar{e} R f R=f R \bar{e} R$, then $f R=\bar{e} R$.

PROOF. Since

$$
\bar{e} R \leq \oplus e R \oplus f R, \quad \bar{e} R=\bar{e}^{2} R \leq \bar{e} f R \leq \bar{e} R f R=f R \bar{e} R \leq f R,
$$

so that $\bar{e} R \leq f R$. Similarly, $f R \leq \bar{e} R$ so we have equality.

A property of idempotents related to unique complementation is subcommutativity.

DEFINITION 2.7 [R65]. An element $a \in R$ is right subcommutative if $R a \subseteq a R$; it is left subcommutative if $a R \subseteq R a$. The element $a$ is subcommutative if it is both left and right subcommutative. A subset of $R$ is left (right) subcommutative if each of its elements is.

The concept of one-sided subcommutativity frequently occurs in the ring theory literature under different names. For example, Birkenmeier [B83, p. 569] defines an idempotent $e \in E$ to be left (right) semicentral if $R e=e \operatorname{Re}(e R=e R e)$. It is readily seen that left semicentral is equivalent to our right subcommutative.

The importance of subcommutativity in the present context is that if $\alpha \in \operatorname{End}(M)$ is right subcommutative, then for all $\beta \in \operatorname{End}(M), \beta \alpha(M) \subseteq \alpha(M)$, that is, $\alpha(M)$ is fully invariant. Reid [R65, Section 3] gives an example of a noncommutative left subcommutative endomorphism ring. However, we shall show in Section 3 that subcommutative idempotents are actually central.

We show now that an idempotent $e$ is left subcommutative if and only if $e R$ has a unique complement.

Proposition 2.8. Let $e \in E$. The following are equivalent:

(1) e is left subcommutative;

(2) $e R \bar{e}=0$;

(3) $\bar{e}$ is right subcommutative;

(4) eR has a unique complement.

PRoOf. (1) $\Leftrightarrow$ (2) For all $r \in R$, (1) implies that $e r \in R e$, so $e R \bar{e} \subseteq R e \bar{e}=0$. Conversely, for all $r \in R, r=r e+r \bar{e}$, so $e r=e r e+e r \bar{e}=e r e \in R e$.

(2) $\Leftrightarrow$ (3) For all $r \in R, r=e r+\bar{e} r$. Hence (2) implies that $r \bar{e}=\bar{e} r \bar{e} \in \bar{e} R$. Conversely, for all $r \in R, r \bar{e} \in \bar{e} R$, so $e r \bar{e} \in e \bar{e} R=0$.

(2) $\Leftrightarrow$ (4) This is Proposition 2.4.

A related commutativity condition on rings is that one-sided ideals are two-sided. 
Definition 2.9. A ring is called right (left) duo if every right (left) ideal is twosided.

PROPOSITION 2.10. A ring is right (left) duo if and only if it is right (left) subcommutative.

PROOF. $(\Rightarrow)$ Let $a \in R$. Then $a R$ is a right ideal, which by hypothesis must be also left ideal. Since rings have identity, $a \in a R$ and so $R a \subseteq a R$.

$(\Leftarrow)$ Let $I$ be a right ideal of $R$. In order to check that $I$ is also left ideal, we have to show that for all $a \in R, R a \subseteq I$. But this is immediate, since $R a \subseteq a R \subseteq I$ by right subcommutativity of $a$.

\section{Abelian rings}

Recall that $R$ is called abelian if $E \subseteq Z(R)$. A nonempty subset of $R$ is called commutative if each pair of its elements commute. A surprising result noticed by Lam [Lam95, Exercise 22.3A, with solution] is that $R$ is abelian if and only if $E$ is commutative and, more generally, if and only if every $e \in E$ commutes with the idempotents $f$ for which $e R \cong f R$. Consequently, we have the following theorem.

THEOREM 3.1. Let $R$ be ring with idempotent set $E$. The following statements are equivalent.

(1) E is commutative.

(2) $R$ is abelian.

(3) Every idempotent generated right ideal is uniquely complemented.

(4) E is subcommutative.

PROOF. (1) $\Rightarrow(2)$ By the remark in the previous paragraph, every idempotent is central.

(2) $\Rightarrow$ (3) is an immediate consequence of Proposition 2.8.

(3) $\Rightarrow$ (4) is also an immediate consequence of Proposition 2.8.

(4) $\Rightarrow$ (1) By Proposition 2.8, for all $e \in E, e R \bar{e}=0=\bar{e} R e$. Hence

$$
f e=e f e+\bar{e} f e=e f e=e f e+e f \bar{e}=e f .
$$

This concludes the proof.

EXAMPLE 3.2. For an example of a ring which is abelian but noncommutative, let $\left\{D_{i}: i \in I\right\}$ be a set of nonisomorphic division rings indexed by an arbitrary set $I$, not all of which are fields. Let $R=\prod_{i \in I} D_{i}$ and consider $R$ as a right $R$-module. It is clear that the only idempotents in $R$ are the sequences $\left(a_{i}: i \in I\right)$ where each $a_{i}$ is either the identity or the zero of $D_{i}$. Thus all right ideals of $R$ are uniquely complemented, so $R$ is an abelian ring.

Abelian rings have many properties in common with commutative rings; for example, we have seen that complements and idempotent generators of idempotent generated ideals are unique. Another such property is the following proposition. 
PROposition 3.3. If $R$ is abelian then $E$ is a Boolean algebra with respect to the natural order ef $\leq e$.

PROOF. For all $e$ and $f \in E$, ef is an idempotent satisfying $e f \leq e$ and $e f \leq f$. Furthermore, if $g \in E$ satisfies $g \leq e$ and $g \leq f$, then $g \leq e f$. Hence $E$ contains $e f=\inf \{e, f\}$.

Next, $e+f-e f$ is an idempotent satisfying $e \leq e+f-e f$ and $f \leq e+f-e f$. If $g \in E$ satisfies $e \leq g$ and $f \leq g$, then $e+f-e f \leq g$. Hence $E$ contains the element $e+f-e f=\sup \{e, f\}$.

Each $e \in E$ has complement $\bar{e} \in E$, and for $e, f$ and $g \in E, \quad(e+f-e f) g=$ $e g+f g-e g f g$ while

$$
e f+g-e f g=(e+g-e g)(f+g-f g),
$$

so $E$ is a distributive lattice.

The following corollary is immediate.

Corollary 3.4. Let $R$ be an abelian ring and let $\mathcal{L}$ be the set of idempotent generated right ideals of $R$, ordered by inclusion. Then $\mathcal{L}$ is a Boolean algebra and the map $e \mapsto e R$ from $E$ to $\mathcal{L}$ is a Boolean algebra isomorphism.

Other related properties are that abelian rings are Dedekind finite and products and intersections of idempotent generated right ideals coincide. Recall that $R$ is Dedekind finite if for all $a, b \in R, a b=1$ implies that $b a=1$.

Proposition 3.5. If $R$ is abelian, the $R$ is Dedekind finite. The converse is false.

Proof. Let $a b=1$. Then $b a=b(a b) a=(b a)(b a)$ is an idempotent. Since $R$ is abelian, $b a R(1-b a)=0$ so in particular, $b a b(1-b a)=0$. Hence

$$
1-b a=(a b)^{2}(1-b a)=0
$$

so $b a=1$.

To see that the converse is false, let $R$ be the $2 \times 2$ matrix ring over a feld.

Proposition 3.6. Let $R$ be an abelian ring and let $e, f \in E$. Then ef $R=e R f R=$ $e R \cap f R$.

PRoOF. Clearly efR $\leq e R f R \leq e R \cap f R$. Conversely, let $x=e r=f s \in e R \cap f R$. Then $x=e^{2} r=e f s \in e f R$.

Finally, we consider the semicommutativity of $E$. A subset $X$ of a $\operatorname{ring} R$ is called semicommutative [CN08, Definition 1.2] if for all $a, b \in X, a b=0$ implies that $a R b=0$.

PROPOSITION 3.7. E is semicommutative if and only if $R$ is abelian.

Proof. For all $e \in E, e \bar{e}=0$ so that $e R \bar{e}=0$. Hence by Proposition $2.4 e R$ is uniquely complemented so $e$ is central.

Conversely, if $e$ is central and $e f=0$, then obviously $e R f=0$. 


\section{Modules with abelian endomorphism rings}

We now show how the results of the previous section apply to endomorphism rings of modules. Throughout, $R$ is a unital ring and all modules are unital right $R$-modules. Homomorphisms are written on the left of their arguments. To avoid confusion, we write $\mathcal{E}$ for $E(\operatorname{End}(M))$, the set of idempotents in $\operatorname{End}(M)$. A module $M$ is an endabelian module if its endomorphism ring $\operatorname{End}(M)$ is abelian.

REMARK 4.1. Since left multiplications from $R$ are in $\operatorname{End}(M)$, if $M$ is an endabelian module then $R$ is an abelian ring.

For a direct decomposition $J \oplus K$ of $M$, the associated projections $\varepsilon_{J}$ and $\varepsilon_{K}$ are the uniquely determined idempotents in $\mathcal{E}$ for which $J=\operatorname{im} \varepsilon_{J}=\operatorname{ker} \varepsilon_{K}$ and $K=\operatorname{ker} \varepsilon_{J}=\operatorname{im} \varepsilon_{K}$. Thus $\varepsilon_{K}=\overline{\varepsilon_{J}}$. We show that summands of $M$ are uniquely determined by right ideals generated by idempotents and vice versa.

Lemma 4.2. Let $M=J \oplus K$. Then $\varepsilon_{J} \operatorname{End}(M)=\{f \in \operatorname{End}(M): f(M) \subseteq J\}$.

Conversely, let $\varepsilon \in \mathcal{E}$ and let $J=\operatorname{trace}(\varepsilon \operatorname{End}(M))$, the submodule of $M$ generated by $\{\varepsilon f(M): f \in \operatorname{End}(M)\}$. Then $M=J \oplus K, \varepsilon_{J}=\varepsilon$ and $\varepsilon_{K}=\bar{\varepsilon}$.

Proof. (1) Clearly for all $f \in \operatorname{End}(M), \varepsilon_{J} f$ maps $M$ into $J$. Also, if $f \in \operatorname{End}(M)$ maps $M$ into $J$, then $f=\varepsilon_{J} f \in \varepsilon_{J} \operatorname{End}(M)$.

(2) Let $m \in M$. Then $m=\varepsilon m+(m-\varepsilon m)$ where $\varepsilon m \in \operatorname{trace}(\varepsilon \operatorname{End}(M))$ and $m-\varepsilon m \in \operatorname{ker} \varepsilon=\bar{\varepsilon}(M)$.

Lemma 4.3. Let $M=J \oplus K$. The following are equivalent:

(1) $J$ is uniquely complemented in $M$;

(2) $\varepsilon_{J} \operatorname{End}(M)$ is uniquely complemented in $\operatorname{End}(M)$, considered as a right $\operatorname{End}(M)$ module;

(3) $\varepsilon_{J} \operatorname{End}(M) \varepsilon_{K}=0$;

(4) $K$ is fully invariant in $M$.

PROOF. (1) $\Leftrightarrow$ (2) By Lemma 4.2, for any submodule $L$ of $M, L$ is a complementary summand for $J$ if and only if $\varepsilon_{L} \operatorname{End}(M)$ is a complementary summand for $\varepsilon_{J} \operatorname{End}(M)$. Hence the uniqueness of $K$ implies the uniqueness of $\varepsilon_{K} \operatorname{End}(M)$ and vice versa.

(2) $\Leftrightarrow$ (3) By Proposition 2.4, $\varepsilon_{J}$ End $(M)$ is uniquely complemented in End $(M)$ if and only if $\varepsilon_{J} \operatorname{End}(M) \varepsilon_{K}=0$.

(3) $\Leftrightarrow$ (4) $\operatorname{Since} \operatorname{End}(M)=\operatorname{Hom}(J, J) \oplus \operatorname{Hom}(J, K) \oplus \operatorname{Hom}(K, J) \operatorname{Hom}(K, K)$, $K$ is fully invariant if and only if $\operatorname{Hom}(K, J)=0$. But since every $f \in \operatorname{Hom}(K, J)$ satisfies $f=\varepsilon_{J} g \varepsilon_{K}$ for some $g \in \operatorname{End}(M), K$ is fully invariant if and only if $\varepsilon_{J} \operatorname{End}(M) \varepsilon_{K}=0$.

THEOREM 4.4. Let $M$ be an $R$-module. The following are equivalent:

(1) all direct summands of $M$ are uniquely complemented;

(2) $M$ is an endabelian module;

(3) all direct summands of $M$ are fully invariant. 
PROOF. In view of Lemma 4.3, it suffices to show that $\operatorname{End}(M)$ is abelian if and only if for all $\varepsilon \in \mathcal{E}, \varepsilon \operatorname{End}(M)$ is uniquely complemented. But this precisely the content of Theorem 3.1.

In the theory of commutative endomorphism rings of mixed abelian groups, a major role is played by groups $G$ of the form

$$
\bigoplus_{p \in S} G_{p} \leq G \leq \prod_{p \in S} G_{p}
$$

where $S$ is an infinite set of primes, for all $p \in S, G_{p} \cong \mathbb{Z}\left(p^{k_{p}}\right)$ with $k_{p}$ a positive integer or $\infty$, and $G$ is $S$-pure in $\prod_{p \in S} G_{p}$.

Such groups have endomorphism rings of the form

$$
\bigoplus_{p \in S} \operatorname{End}\left(G_{p}\right) \subseteq \operatorname{End}(G) \subseteq \prod_{p \in S} \operatorname{End}\left(G_{p}\right)
$$

where $\operatorname{End}\left(G_{p}\right) \cong \mathbb{Z}\left(p^{k_{p}}\right)$ if $k_{p}$ is finite and $\operatorname{End}\left(G_{p}\right) \cong \mathbb{Z}_{p}$, the ring of $p$-adic integers, if $k_{p}=\infty$.

In particular, $\operatorname{End}(G)$ is commutative. This result appears, for example, in [KMT03, S73, SzSz51].

In the torsion-free context, a similar result is obtained by replacing the set $S$ of primes by a set $T$ of incomparable idempotent types $\tau$ (that is, subrings of $\mathbb{Q}$ ), the groups $G_{p}$ by the types $\tau \in T$ and the $S$-purity condition by

$$
\operatorname{Hom}\left(G / \bigoplus_{\tau \in T} \tau, G\right)=0 .
$$

In both cases, the commutativity of $\operatorname{End}(G)$ follows from the commutativity of each $\operatorname{End}\left(G_{p}\right)$ and $\operatorname{End}(\tau)$, together with full invariance of the indecomposable summands $G_{p}$ and $\tau$. However, the structure theorem can be generalized as follows without any commutativity assumption on the endomorphism rings of the summands.

DEFINITION 4.5. A ring $R$ with idempotent set $E$ is called nearly commutative if:

(1) $R$ is abelian;

(2) for every $0 \neq a \in R$, there is a primitive idempotent $e \in E$ such that $e a \neq 0$.

REMARK 4.6.

(a) We say that a module $M$ is an NC module if $\operatorname{End}(M)$ is nearly commutative.

(b) Condition (2) implies that every nonzero idempotent in $E$ dominates a primitive idempotent, that is, for all $0 \neq e \in E$ there is a primitive idempotent $f$ such that $f e=f=e f$.

(c) The abelian group example above shows that (2) may hold even when the dcc on idempotents does not.

(d) To see that the conditions are independent, note that the ring $\mathbb{Z}(p)^{2}$ satisfies (2) but not (1), and the semigroup ring $\mathbb{Z}[S]$, where $S$ is the semigroup $\left\{s_{i}: i \in \mathbb{Z}\right\}$ with $s_{i} s_{j}=s_{\min (i, j)}$, satisfies (1) but not (2). 
The following theorem describes the structure of nearly commutative rings.

THEOREM 4.7. Let $R$ be a nearly commutative ring, and let $F$ be a maximal orthogonal set of primitive idempotents in $R$. Then $R$ is a subdirect product of $\{e R: e \in F\}$ containing $\bigoplus_{e \in F} e R$. That is to say,

$$
\bigoplus_{e \in F} e R \leq R \leq \prod_{e \in F} e R
$$

PROOF. First note that condition (2) of near commutativity implies that $R$ has nonzero primitive idempotents and Zorn's lemma implies that maximal orthogonal sets of primitive idempotents exist in $R$.

Let $F$ be a maximal orthogonal set of primitive idempotents in $R$, and define $\theta: R \rightarrow \prod_{e \in F} e R$ by $x \mapsto(e x)$. It is clear that:

(1) $\theta$ is a module homomorphism;

(2) $\theta$ preserves multiplication;

(3) the image of $\theta$ contains all $e R, e \in F$ and hence $\bigoplus_{e \in F} e R$.

Let $K=\operatorname{ker}(\theta)$ and let $x \in K$. By condition (2), if $x \neq 0$, then there is a primitive idempotent $0 \neq f \in R$ such that $f x \neq 0$ and hence $f \notin F$. But for all $e \in F$, either $f e=e=f$ or $f e=0$. Thus $f e=0$ for all $e \in F$. Therefore $F \cup\{f\}$ is an orthogonal set of primitive idempotents strictly containing $F$, a contradiction.

Hence $K=0$ so that $\theta$ is an embedding.

COROLLARY 4.8. If $R$ is a nearly commutative ring, then $R$ is a unique subdirect product of indecomposable ideals.

\section{REMARK 4.9.}

(1) It is easy to find countable reduced torsion-free rings satisfying the hypotheses of Theorem 4.7. For example, take any countable poset $P$ satisfying the descending chain condition, let $S$ be the semigroup on the set $P$ with operation $x y=\min \{x, y\}$ and let $R$ be the semigroup ring $\mathbb{Z}[S]$. Then $R$ satisfies Theorem 4.7, and by Corner's theorem there is a torsion-free abelian group $G$ with $\operatorname{End}(G) \cong R$.

(2) The abelian condition may be weakened to primitive idempotents being central.

Recall that a family $\mathcal{M}$ of modules is rigid if for all $L, N \in \mathcal{M}, \operatorname{Hom}(L, N)=0$. Our main structure theorem is the following.

THEOREM 4.10. Let $M$ be an R-module. $M$ is NC if and only if there is a rigid family $\mathcal{M}$ of indecomposable $R$-modules such that

$$
\bigoplus_{N \in \mathcal{M}} N \leq M \leq \prod_{N \in \mathcal{M}} N \text { and } \operatorname{Hom}\left(M /\left(\bigoplus_{N \in \mathcal{M}} N\right), M\right)=0 .
$$

PROOF. $(\Rightarrow)$ Since End $(M)$ is a subdirect product of indecomposable ideals generated by primitive idempotents, $M$ contains a unique rigid maximal family $\mathcal{M}$ of fully invariant indecomposable summands and hence their direct sum $\bigoplus_{N \in \mathcal{M}} N$. 
The projections of $M$ onto its summands $N$ induce a homomorphism of $M$ into $\prod_{N \in \mathcal{M}} N$. Since this homomorphism has kernel zero, $\operatorname{Hom}(M /(\oplus N), M)=0$.

$(\Leftarrow)$ Since each $N$ is fully invariant in $M$, each $\operatorname{End}(N)$ is an ideal summand in $\operatorname{End}(M)$ and their sum is direct. The homomorphism $\Theta: \operatorname{End}(M) \rightarrow$ $\prod_{N \in \mathcal{M}} N$ defined by $f \mapsto\left(\varepsilon_{N} f\right)$ has kernel $\{f \in \operatorname{End}(M): f(\oplus N)=0\}$. But since $\operatorname{Hom}_{R}(M /(\oplus N), M)=0$ this kernel is 0 , so $\Theta$ is injective.

\section{Fully invariant submodules of $M$}

We are concerned with properties of $\operatorname{End}(M)$ which imply that certain classes of submodules of $M$ are fully invariant. For example, we have seen that direct summands are fully invariant if and only if $M$ is an endabelian module, which implies that $R$ is an abelian ring. With this in mind, we define $M$ to be stable if all endomorphic images are fully invariant, and substable if all kernels of endomorphisms are fully invariant. We noted in the paragraph following Definition 2.7 that if $\alpha \in \operatorname{End}(M)$ is right subcommutative, then $\alpha(M)$ is fully invariant, so if $\operatorname{End}(M)$ is right subcommutative, then $M$ is stable.

It is clear that if $\operatorname{End}(M)$ is commutative then $M$ is stable and substable. It has long been known that the converse is false, even for abelian groups, but in order to find a counterexample one must construct a torsion-free countable reduced ring which is subcommutative but not commutative and then invoke Corner's theorem to show that it is an endomorphism ring. This was done independently by Orsatti and Reid in 1965 [KMT03, p. 123]. Other examples have been constructed since, but they all rely on the same principle.

Our aim here is to elucidate the relations between (sub)stability of $M$ and subcommutativity of $\operatorname{End}(M)$.

Proposition 5.1. Let $\alpha \in \operatorname{End}(M)$.

(1) If $\alpha$ is left subcommutative then $\operatorname{ker} \alpha$ is fully invariant.

(2) If $\operatorname{im} \alpha$ is a summand of $M$ and $\operatorname{ker} \alpha$ is fully invariant, then $\alpha$ is left subcommutative.

Proof. (1) Let $\beta \in \operatorname{End}(M)$. There exists $\gamma \in \operatorname{End}(M)$ such that $\alpha \beta=\gamma \alpha$. Let $a \in \operatorname{ker} \alpha$, so $\alpha \beta(a)=\gamma \alpha(a)=0$. Hence $\beta a \in \operatorname{ker} \alpha$.

(2) Let $\alpha, \beta \in \operatorname{End}(M)$. Suppose that im $\alpha$ is a summand of $M$. Define $\gamma \in \operatorname{End}(G)$ by $\gamma(\alpha a)=\alpha \beta a$ on $\operatorname{im} \alpha$ and $\gamma=0$ on a complement. $\gamma$ is well defined since if $\alpha a=\alpha b$ then $a-b \in \operatorname{ker} \alpha$ so $\beta a-\beta b \in \operatorname{ker} \alpha$ and hence $\alpha \beta a=\alpha \beta b$.

It follows that $\alpha \beta=\gamma \beta$ as required.

Corollary 5.2. If $\alpha \in \operatorname{End}(M)$ is idempotent or an epimorphism, then $\alpha$ is left subcommutative if and only if $\operatorname{ker} \alpha$ is fully invariant.

On the other hand, we have the following proposition.

Proposition 5.3. Let $\alpha \in \operatorname{End}(M)$.

(1) If $\alpha$ is right subcommutative then $\operatorname{im} \alpha$ is fully invariant. 
(2) If $\alpha$ is a monomorphism and $\operatorname{im} \alpha$ is fully invariant, then $\alpha$ is right subcommutative.

Proof. (1) Let $\beta \in \operatorname{End}(M)$. There exists $\gamma \in \operatorname{End}(M)$ such that $\beta \alpha=\alpha \gamma$. Let $a \in \operatorname{im} \alpha$, say $a=\alpha b$. Then $\beta a=\beta \alpha b=\alpha \gamma(a) \in \operatorname{im} \alpha$.

(2) Suppose $\alpha$ is monic. Since im $\alpha$ is fully invariant, for every $a \in M$, there is a unique $b$ in the preimage $\alpha^{-1}(\beta \alpha(a)) \neq \varnothing$. Let $\gamma$ be defined by $\gamma(a)=b$. It follows that $\gamma \in \operatorname{End}(M)$ and $\beta \alpha=\alpha \gamma$ as required.

COROLlaRY 5.4. If $\alpha \in \operatorname{End}(M)$ is idempotent or a monomorphism, then $\alpha$ is right subcommutative if and only if im $\alpha$ is fully invariant.

Corollary 5.5. Let $M$ be an R-module.

(1) If $\operatorname{End}(M)$ is right subcommutative then $M$ is stable. The converse holds if every endomorphism is monic.

(2) If $\operatorname{End}(M)$ is left subcommutative then $M$ is substable. The converse holds if every endomorphism is epic.

We have seen that if $\varepsilon \in \operatorname{End}(M)$ is a central idempotent, then $M=\operatorname{im} \varepsilon \oplus \operatorname{ker} \varepsilon$ where each summand is fully invariant in $M$ and hence uniquely complemented. We now address the converse of this result, that is, if $M$ is the direct sum of two fully invariant submodules, are they the image and kernel of a central idempotent in $\operatorname{End}(M)$ ? More generally, if $H$ is a fully invariant submodule of $M$, when is $H$ a summand with a fully invariant complement?

We first consider the relationship between fully invariant submodules of $M$ and (two-sided) ideals of $\operatorname{End}(M)$. Let $\mathcal{H}$ be the set of fully invariant submodules of $M$ and $\mathcal{I}$ the set of ideals of $\operatorname{End}(M)$. It is routine to check that $\mathcal{H}$ and $\mathcal{I}$ are complete lattices under inclusion. Denote by $\mathcal{H}^{\text {op }}$ the opposite lattice of $\mathcal{H}$.

For all $H \in \mathcal{H}$, let

$$
H^{\prime}=\operatorname{Ann}(H)=\{\alpha \in \operatorname{End}(M): \alpha(H)=0\}
$$

and

$$
H^{*}=\operatorname{Ann}(M / H)=\{\alpha \in \operatorname{End}(M): \alpha(M) \subseteq H\} .
$$

It is routine to check that $H^{\prime}$ and $H^{*} \in \mathcal{I}$. Similarly, for all $I \in \mathcal{I}$, let $I^{\prime}=\{a \in M$ : $I a=0\}$ and let $I^{*}=I M$, the submodule of $M$ generated by $\{\alpha(a): \alpha \in I$ and $a \in M\}$, so that $I^{\prime}$ and $I^{*} \in \mathcal{H}$.

It was shown in [AS04] that the mappings $\Theta^{\prime}: \mathcal{H} \rightarrow \mathcal{I}$ defined by $H \mapsto H^{\prime}$ and $\Theta^{*}: \mathcal{H}^{\mathrm{op}} \rightarrow \mathcal{I}$ defined by $H \mapsto H^{*}$ are Galois correspondences, with inverses defined by $I \mapsto I^{\prime}$ and $I \mapsto I^{*}$ respectively. In particular, for all $H \in \mathcal{H}, H \leq H^{\prime \prime}$ and $H^{* *} \leq H$ and, for all $I \in \mathcal{I}, I \leq I^{\prime \prime}$ and $I \leq I^{* *}$.

An $H \in \mathcal{H}$ is called '-closed if $H=H^{\prime \prime}$ and ${ }^{*}$-closed if $H=H^{* *}$. Similarly, $I \in \mathcal{I}$ is ' ${ }^{-}$-closed if $I=I^{\prime \prime}$ and ${ }^{*}$-closed if $I=I^{* *}$. It follows that $H$ is ' ${ }^{\prime}$-closed if and only if $H=I^{\prime}$ for some ideal $I \in \mathcal{I}, \quad I \in \mathcal{I}$ is '-closed if and only if $I=H^{\prime}$ for some $H \in \mathcal{H}, \quad H$ is ${ }^{*}$-closed if and only if $H=I^{*}$ for some $I \in \mathcal{I}$, and $I$ is ${ }^{*}$-closed if and only if $I=H^{*}$ for some $H \in \mathcal{H}$. 
We have seen that if $I \in \mathcal{I}$ is a summand of $\operatorname{End}(M)$ then it is generated by a central idempotent and is uniquely complemented, while it is clear that if $H \in \mathcal{H}$ is a summand of $M$, then it is the image of a central idempotent and is uniquely complemented.

We now describe conditions on $I \in \mathcal{I}$ and on $H \in \mathcal{H}$ which ensure that they are summands.

Proposition 5.6. Let $I \in \mathcal{I}$. The following conditions are equivalent:

(1) $I$ is a summand of $\operatorname{End}(M)$;

(2) $I$ is '-closed, $I \cap\left(I^{\prime}\right)^{*}=0$ and every endomorphism of $I^{\prime}$ extends to an endomorphism of $M$;

(3) I is *-closed, $I \cap\left(I^{*}\right)^{\prime}=0$ and every endomorphism of $M / I^{*}$ lifts to an endomorphism of $M$.

If these conditions are satisfied, then $M=I^{\prime} \oplus I^{*}$.

ProOF. (1) $\Leftrightarrow(2)$ Suppose that $I$ is a summand of $\operatorname{End}(M)$, say

$$
\operatorname{End}(M)=\varepsilon \operatorname{End}(M) \oplus \bar{\varepsilon} \operatorname{End}(M) \quad \text { where } I=\varepsilon \operatorname{End}(M)
$$

for some central idempotent $\varepsilon$. Then $I^{\prime}=\bar{\varepsilon}(M)=H$, say, and $H^{\prime}=I$, so $I=I^{\prime \prime}$. Hence $H=\operatorname{ker} \varepsilon=I^{\prime}$ is a summand of $M$ so that $I \cap\left(I^{\prime}\right)^{*}=0$ and every endomorphism of $H$ extends to $\operatorname{End}(M)$.

Conversely, [AS04, Proposition 2.4] implies that if $I \in \mathcal{I}$ with $I \cap\left(I^{\prime}\right)^{*}=0$, then there is a short exact sequence

$$
I^{\prime \prime} \longmapsto \operatorname{End}(M) \stackrel{\eta}{\rightarrow} \operatorname{End}(M)^{H}
$$

where the latter ring is the set of endomorphisms of $H$ which extend to endomorphisms of $M, \eta$ being the restriction map. If every such endomorphism extends, then the sequence splits so

$$
\operatorname{End}(M) \cong I \oplus \operatorname{End}(H)
$$

as a direct sum of ideals.

$(1) \Leftrightarrow(3)$ The proof is similar, so only sketched. If $I$ is a summand of $\operatorname{End}(M)$, with central idempotent generator $\varepsilon$, let $H=\varepsilon(M)=I^{*}$. Then $I=H^{*}$ and $M=H \oplus I^{\prime}$, so that $I=I^{* *}$ and $I \cap\left(I^{*}\right)^{\prime}=0$. Since $H$ is a summand of $M$, every endomorphism of $M / H=I^{\prime}$ lifts to $\operatorname{End}(M)$.

Conversely, [AS04, Proposition 2.4] implies that the hypotheses force $\operatorname{End}(M)=$ $H^{\prime} \oplus H^{*}=\left(I^{*}\right)^{\prime} \oplus I$.

Proposition 5.7. Let $H \in \mathcal{H}$. The following conditions are equivalent:

(1) $H$ is a summand of $M$;

(2) $H$ is ' -closed, $H^{\prime} \cap H^{*}=0$ and every endomorphism of $H$ extends to an endomorphism of $M$;

(3) $\quad H$ is ${ }^{*}$-closed, $H^{*} \cap H^{\prime}=0$ and every endomorphism of $M / H$ lifts to an endomorphism of $M$.

If these conditions are satisfied, then $\operatorname{End}(M)=H^{\prime} \oplus H^{*}$. 
ProOF. (1) $\Leftrightarrow(2)$ Suppose that $M=H \oplus K$, and let $\varepsilon$ be the corresponding projection onto $K$. Since $H$ is fully invariant, $\bar{\varepsilon}$ and its complement $\varepsilon$ are central idempotents in $\operatorname{End}(M)$. Let $I=\varepsilon \operatorname{End}(M)$ and $L=\bar{\varepsilon} \operatorname{End}(M)$. Then $H=I^{\prime}$ is '-closed and $L=H^{*}$ so that $H^{\prime} \cap H^{*}=0$. Clearly every endomorphism of $H$ extends to an endomorphism of $M$.

Conversely, the hypotheses and [AS04, Proposition 2.4] imply that

$$
\operatorname{End}(M)=H^{\prime} \oplus H^{*}
$$

with central idempotent generators $\varepsilon$ and $\bar{\varepsilon}$ so that $H=\operatorname{ker} \varepsilon$ is a summand with complement im $\varepsilon$.

(1) $\Leftrightarrow(3)$ The proof in both directions is similar.

The criteria of Propositions 5.6 and 5.7 simplify if $\operatorname{End}(M)$ is abelian since in that case, by Theorem 4.4, all direct summands are fully invariant. The following theorem is immediate.

THEOREM 5.8. Let $R$ be $a$ ring and $M$ an endabelian $R$-module. Let $H$ be $a$ submodule of $M$ and $I$ an ideal of $\operatorname{End}(M)$.

(1) The following are equivalent:

(a) $H$ is a direct summand of $M$;

(b) $H$ is fully invariant and '-closed, $H^{\prime} \cap H^{*}=0$ and every endomorphism of $H$ extends to an endomorphism of $M$;

(c) $H$ is fully invariant and ${ }^{*}$-closed, $H^{*} \cap H^{\prime}=0$ and every endomorphism of $M / H$ lifts to an endomorphism of $M$.

In that case, $\operatorname{End}(M)=H^{\prime} \oplus H^{*}$.

(2) The following are equivalent:

(a) I is a direct summand of $\operatorname{End}(M)$;

(b) $I$ is '-closed, $I \cap\left(I^{\prime}\right)^{*}=0$ and every endomorphism of $I^{\prime}$ extends to an endomorphism of $M$;

(c) I is *-closed, $I \cap\left(I^{*}\right)^{\prime}=0$ and every endomorphism of $M / I^{*}$ lifts to an endomorphism of $M$.

In that case, $M=I^{\prime} \oplus I^{*}$.

\section{References}

[AS04] R. Abraham and P. Schultz, 'Additive Galois theory of modules', in: Rings, Modules, Algebras and Abelian Groups, Lecture Notes in Pure and Applied Mathematics, 236 (Marcel Dekker, New York, 2004), pp. 1-12.

[B83] G. F. Birkenmeier, 'Idempotents and completely semiprime ideals', Comm. Algebra 11 (1983), 567-580.

[CN08] V. Camillo and P. P. Nielsen, 'McCoy rings and zero-divisors', J. Pure Appl. Algebra 212 (2008), 599-615.

[F73] L. Fuchs, Infinite Abelian Groups, Vols. I and II (Academic Press, New York, 1970), 1973.

[KMT03] P. A. Krylov, A. V. Mikhalev and A. A. Tuganbaev, Endomorphism Rings of Abelian Groups, Algebras and Applications, 2 (Kluwer Academic Publishers, Dordrecht, 2003).

[Lam95] T. Y. Lam, Exercises in Classical Ring Theory, Problem Books in Mathematics (Springer, New York, 1995). 
[R65] J. D. Reid, 'On subcommutative rings', Acta Math, Acad. Sci. Hungar. 16 (1965), 23-26.

[S73] P. Schultz, 'The endomorphism ring of the additive group of a ring', J. Aust. Math. Soc. 15(1) (1973), 60-69.

[SzSz51] T. Szele and J. Szendrei, 'On Abelian groups with commutative endomorphism rings', Acta Math. Acad. Sci. Hungar. 2 (1951), 309-324.

GRIGORE CĂLUGĂREANU, Babeş-Bolyai University Cluj-Napoca,

Faculty of Mathematics and Computer Science, Str. Mihail Kogălniceanu nr. 1, RO-400084 Cluj-Napoca, Romania

e-mail: calu@math.ubbcluj.ro

PHILL SCHULTZ, School of Mathematics and Statistics, University of Western Australia, Nedlands, WA 6009, Australia

e-mail: schultz@maths.uwa.edu.au 